\title{
Interacting Polyelectrolyte Brushes Grafted in Two Bilayers: Molecular Dynamics Simulations
}

\author{
Nourdine Hadrioui, Khalid Elhasnaoui, Abdelwahad Maarouf", Tarik ELhafi, Hamid Ridouane
}

LPPPC, Sciences Faculty Ben M'sik, Hassan II University, Casablanca, Morocco

Email address:

Abdelwahad.maarouf@gmail.com (A. Maarouf).

\section{To cite this article:}

Nourdine Hadrioui, Khalid Elhasnaoui, Abdelwahad Maarouf, Tarik ELhafi, Hamid Ridouane. Interacting Polyelectrolyte Brushes Grafted in Two Bilayers: Molecular Dynamics Simulations. American Journal of Physics and Applications. Vol. 4, No. 2, 2016, pp. 20-26. doi: 10.11648/j.ajpa.20160402.11

\begin{abstract}
Using molecular dynamics simulations, we study interacting polyelectrolyte brushes that are grafted to two parallel surfaces (quasi-Planar Membrane). The interactions between brushes are important, for instance, in stabilization of dispersions against flocculation. We simulate the relative shear motion of both neutral and polyelectrolyte end-grafted polymer brushes. The flexible neutral polymer brush is treated as a bead-spring model, and the polyelectrolyte brush is treated the same way except that each bead is charged and there are counter ions present to neutralize the charge. We investigate the friction coefficient, monomer density, and brush penetration for the two kinds of brushes with both the same grafting density and the same normal force under good solvent conditions.
\end{abstract}

Keywords: Molecular Dynamics Simulation, Aqueous Solution, WCA Potential, Membrane, Adhesion

\section{Introduction}

Adhesion of membranes and vesicles has attracted considerable experimental and theoretical interest because of its prime importance to many bio-cellular processes $[1,2]$. Theoretical treatments of membranes composed of single component lipid bilayers have revealed that generic interactions such as van der Waals, electrostatic or hydration interactions govern the adhesive properties of interacting membranes. It is also worthwhile to mention that related phenomena are found in unbinding transition of nearly flat membranes [3] or adhesion of vesicles to surfaces [4].

In addition to general non-specific interactions mentioned above, it is known from the works of Bell and coworkers [5, 6] as well as others [7], that highly specific molecular interactions play an essential role in biological adhesion. This interaction acts between complementary pairs of proteins such as ligand and receptor, or antibody and antigen. A wellstudied example of such coupled systems is the biotin-avidin complex. The avidin molecule has four biotin binding sites, two on each side, and forms a five-molecule biotin-avidinbiotin complex. The resulting specific interaction is highly local and short-ranged.

Currently, the adhesion of two adjacent plasma membranes is provided by bound pairs of such adhesion macromolecules which form bridges between the membranes. We distinguish three types of adhesion depending on the structure of bridges: i) Bolaform-sticker adhesion where each bridge molecule consists of a single sticker having two sticky ends. One sticker end is anchored to one membrane while the other end is adhering directly to the second membrane. ii) Homophilic-sticker adhesion where the bridges are formed by two stickers of the same type. Each sticker is anchored on one of the membranes, while their free ends bind together to form the bridge. iii) Lock-and key adhesion where the bridges consist of two different stickers forming a ligandreceptor type bond. This case represents an asymmetric adhesion due to the lack of symmetry between the ligand and receptor.

A model system for these complex interactions is provided by systems containing lipid bilayers and polymers brush. From the physical point of view, polymer brush consists of polymer chains densely grafted to a membrane surface, which is immersed in a solvent. The chains stretch from membranes and repel each other. The balance between elasticity and repulsion of the chains generates completely different conformations and properties than for isolated chains. Electrostatic interaction involved in polyelectrolyte brushes results in a number of additional physical properties. Mutual repulsion between polymer segments and 
electrostatic forces between charged monomers and counter ions strongly influence the conformation of the polyelectrolyte brush [8,9]. A great deal of theoretical and experimental research has been conducted to investigate the frictional behavior when lateral sliding between two opposing polymer brush membranes occurs $[10,11,12,13$, $14,15,16]$. The normal force and shear force have been well studied experimentally by the surface force apparatus (SFA) both on neutral polymer brush membranes and polyelectrolyte brush membranes. These experiments on neutral polymer brushes showed that the polymer brush surfaces have ultralow friction coefficients when the membrane surfaces are brought into contact [17].

In this work we provide a general phenomenological approach for the adhesion of membranes. Using a molecular dynamics, we simulate the relative shear motion of both neutral and polyelectrolyte end-grafted polymer brushes. The flexible neutral polymer brush is treated as a bead-spring model, and the polyelectrolyte brush is treated the same way except that each bead is charged and there are counter ions present to neutralize the charge. We investigate the friction coefficient, monomer density, and brush penetration for the two kinds of brushes with both the same grafting density and the same normal force under good solvent conditions.
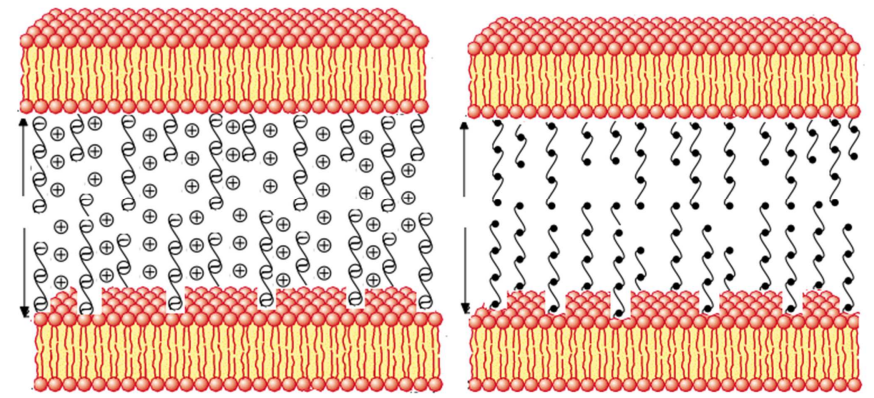

Fig. 1. The schematics of two opposing polymer brushes a neutral brush(on the right) and a charged brush (to the left). Each polymer chain is grafted to its lipid bilayers by its end monomer. Each monomer on charged chains carries one negative charge except for the end-grafted monomer. In addition, explicit monovalent counterions are added to the polyelectrolyte brushes to neutralize the system.

\section{Simulation Details}

Pair-potential used here is that derived by Lennard-Jones (L-J).The repulsive part of the potential (L-J) is Greater than the attractive part at small distances, and inversely at long distances. After this analysis, the potential has the following expression [21]

$$
\mathrm{U}_{\mathrm{LJ}}(\mathrm{r})=\left\{\begin{array}{cl}
-\varepsilon & \mathrm{r} \prec \mathrm{r}_{\mathrm{m}} \\
4 \varepsilon\left[\left(\frac{\sigma}{\mathrm{r}}\right)^{12}-\left(\frac{\sigma}{\mathrm{r}}\right)^{6}\right] & \mathrm{r}_{\mathrm{m}} \prec \mathrm{r} \prec \mathrm{r}_{\mathrm{c}} \\
0 & \mathrm{r} \succ \mathrm{r}_{\mathrm{c}}
\end{array}\right.
$$

where $\sigma$ and $\varepsilon$ are, respectively, the well depth is and distance parameters of the (L-J), $r_{c}$ is the cutoff distance, $r_{m}$ is the distance where the intermolecular potential reaches a minimum.In the (L-J) potential, the short-range repulsion is thought to be dominated by the standard $r^{12}$ law, whereas the long range London attraction varies as $r^{6}$ law. Thus, the repulsive part is set with a power 12 only for convenience, whereas the attractive part has a good theoretical foundation based on three different effects; the dispersion force, the permanent charge distribution and the induced.

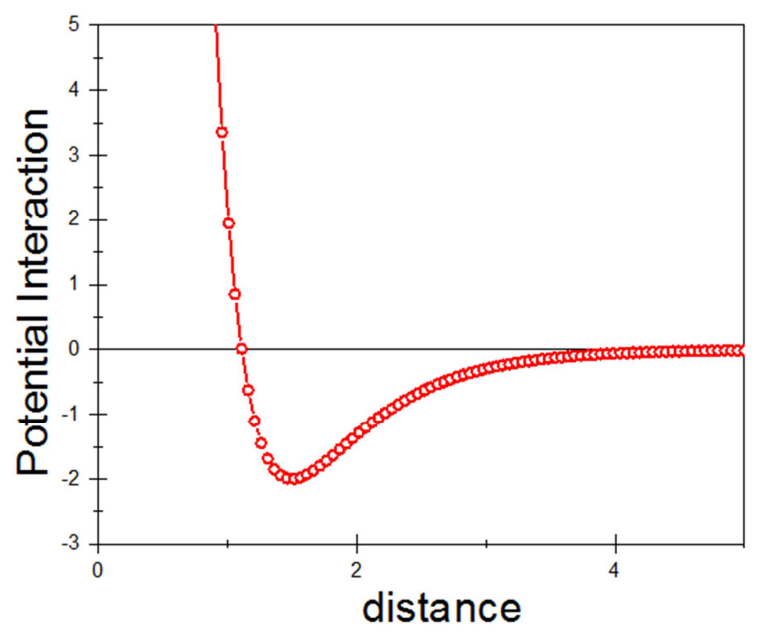

Fig. 2. The Lennard-Jones potential. This potential is repulsive.

for $\mathrm{r} \prec 2^{1 / 6} \sigma$ and attractive for $\mathrm{r} \succ 2^{1 / 6} \sigma$. There is a minimum in the potential at $r=2^{1 / 6} \sigma$.

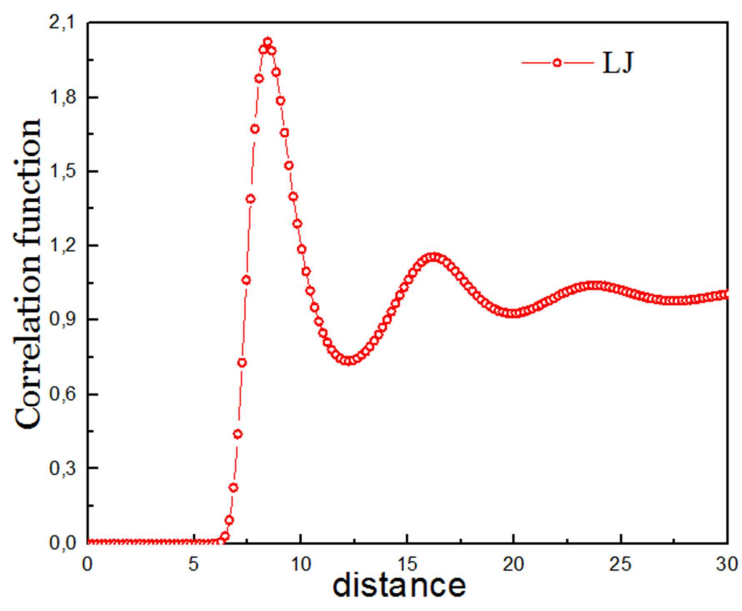

Fig. 3. Correlation function of Lennard-Jones potential.

The first derivative of the expression of (L-J) potential he presented as follows

$$
\frac{\partial}{\partial \mathrm{r}} \mathrm{U}^{\mathrm{LJ}}(\mathrm{r})=12 \varepsilon\left(\sigma^{6} \frac{1}{\mathrm{r}^{7}}-2 \sigma^{12} \frac{1}{\mathrm{r}^{13}}\right),
$$

The position of the potential minimum $r_{m}$ is given as

$$
r_{\mathrm{m}}=2^{1 / 6} \sigma
$$

The expression (1) and (3) in the birth of a new term to the (LJ) potential 


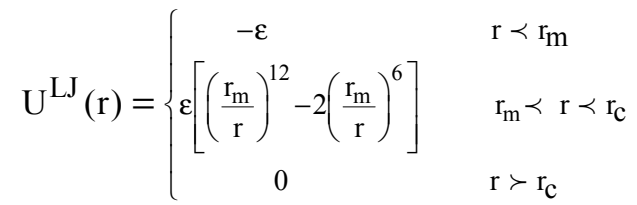

A (L-J) potential with this truncation point is often known as the Weeks-Chandler-Andersen (WCA) potential [19], and it results in purely repulsive interactions. This potential is convenient for computational work because it is shortranged, and therefore computationally undemanding, but still retains the essential physics, i.e. the repulsive (excluded volume) interaction. The truncated and shifted LJ potential is defined as

$$
\mathrm{U}_{\mathrm{WCA}}(\mathrm{r})= \begin{cases}4 \varepsilon\left[\mathrm{U}^{\mathrm{LJ}}(\mathrm{r})-\mathrm{U}^{\mathrm{LJ}}\left(2^{1 / 6} \sigma\right)\right] & \mathrm{r} \prec 2^{1 / 6} \sigma, \\ 0, & \mathrm{r} \succ 2^{1 / 6} \sigma,\end{cases}
$$

where $\varepsilon$ is the (L-J) energy parameter and $\sigma$ the (L-J) length parameter. When using the (L-J) potentials in simulation, it is customary to work in a system of units where $\sigma=1$ and $\varepsilon=1$.

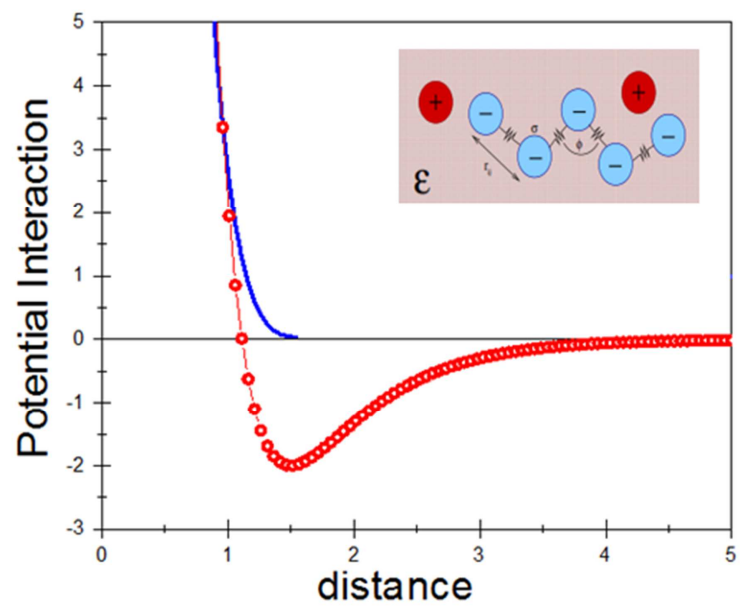

Fig. 4. Comparison of WCA potential (Bleu - solid line) and (L-J) (Red-dotted circles) potential.

The Finitely Extensible Non-linear Elastic (FENE) [20, 21] molecules that we simulate here consist of a linear chain of $\mathrm{N}$ beads interacting via pair potentials. Adjacent beads along the chain interact via a combined FENE spring potential and WCA potential. Beads which are not on the same molecule and beads which are on the same molecule but not adjacent interact just by the WCA potential (5). For two adjacent beads separated by the vector $r$, the FENE contribution to their interaction is,

$$
\mathrm{U}_{\mathrm{FENE}}(\mathrm{r})= \begin{cases}-\frac{1}{2} \mathrm{k} \mathrm{R}_{0}^{2} \ln \left(1-\left(\mathrm{r} / \mathrm{R}_{0}\right)^{2}\right) & \mathrm{r} \prec \mathrm{R}_{0}, \\ \infty, & \mathrm{r} \succ \mathrm{R}_{0},\end{cases}
$$

Where the spring constant $\mathrm{k}=30 \varepsilon_{\mathrm{LJ}} / \sigma^{2}$ and the maximum extent distance $R_{0}=1.5 \sigma$. Logarithmic factor in the FENE potential bears no physical meaning and is strictly for computational advantage.

This potential mimics the stiff and non-penetrable covalent bonds between monomers. The choice of such $L J$ potential and FENE potential parameters prevents bond crossings and yields realistic dynamics of polymer chains [22, 23]. This potential gives rather stiff bond lengths with fluctuations being smaller than $10 \%$. The nonlinearity allows a very efficient mixing of the different vibrational modes.

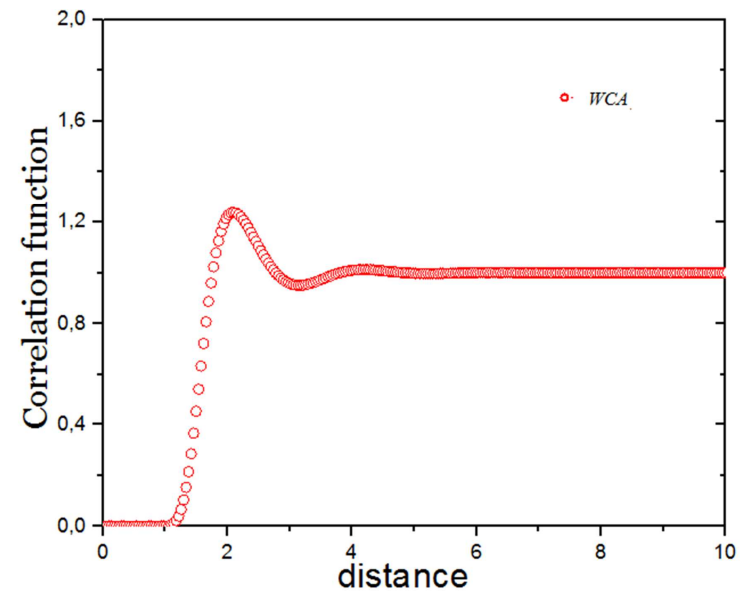

Fig. 5. Correlation function of Weeks-Chandler-Andersen (WCA) potential.

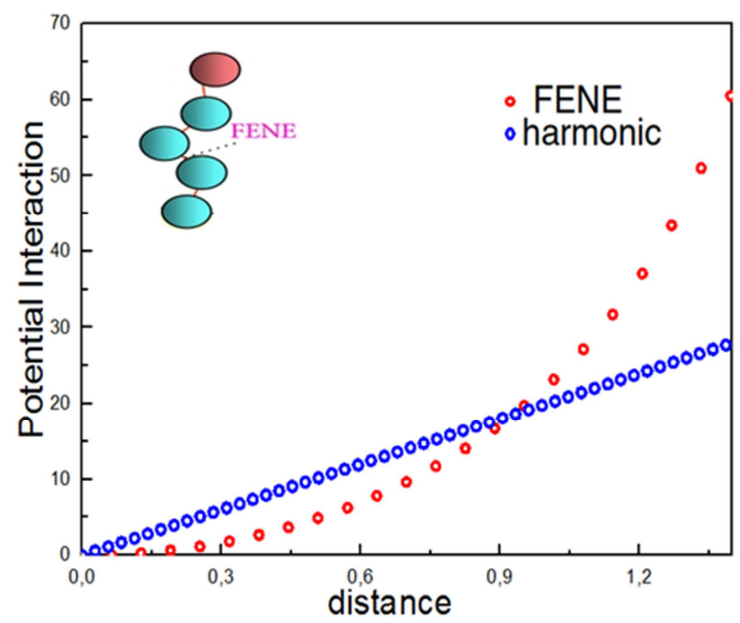

Fig. 6. FENE (finitely extensible nonlinear elastic potential) and harmonic Potential

where $R_{0}$ is a finite extensibility (see Fig.4). The FENE potential is harmonic at its minimum but the bonds cannot be stretched beyond a maximum length determined by $\mathrm{R}_{0}$

The average bond length, found by minimizing $\mathrm{U}_{\mathrm{FENE}}(\mathrm{r})+\mathrm{U}_{\mathrm{LJ}}(\mathrm{r})$, is $\mathrm{b}=0.98 \sigma$.

For polyelectrolyte brushes, the Coulomb interaction between two charged particles is included. Charged particles interact with each other through a solvent medium, which is presumed to be water in the simulation. Then the Coulomb potential between two charged particles $i$ and $j$ in our simulation system is given by

$$
\mathrm{U}_{\text {Coul }}\left(\mathrm{r}_{\mathrm{ij}}\right)=\mathrm{k}_{\mathrm{B}} \mathrm{Tl}_{\mathrm{B}} \frac{\mathrm{q}_{\mathrm{i}} \mathrm{q}_{\mathrm{j}}}{\mathrm{r}_{\mathrm{ij}}}
$$


Where $\mathrm{q}_{\mathrm{i}}=+1$ for the charged chain monomers, and $\mathrm{q}_{\mathrm{i}}=-1$ for the counterions, which are treated explicitly as charged $L J$ particles, $1_{\mathrm{B}}$ is the Bjerrum length which is defined as the length at which the electrostatic energy equals the thermal energy:

$$
\mathrm{l}_{\mathrm{B}}=\frac{\mathrm{e}^{2}}{4 \pi \varepsilon \varepsilon_{0} \mathrm{k}_{\mathrm{B}} \mathrm{T}},
$$

where $\mathrm{e}$ is the unit charge of the interacting particles, and $\varepsilon$ and $\varepsilon_{0}$ are the permittivity of the vacuum and of the solvent, respectively $\left(1_{\mathrm{B}}=7.1 \AA\right.$ in water). Here we restrict ourselves to monovalent species in salt free solution. The solvent is taken into account via the dielectric background, whose properties influence the form (hydrophil or hydrophob) of the interaction potential of the monomers. Unless mentioned the Bjerrum length is always fixed to $1_{B}=3.0 \sigma$.

The particle-particle particle-mesh (PPPM) method implemented in the Large-scale Atomic/Molecular Massively Parallel Simulator (LAMMPS) was used to calculate electrostatic potential between charged particles [24]. All particles except anchored segments interact repulsively with the walls at $z=0$ and $z=L$ with the (L-J) potential,

$$
\mathrm{U}_{\text {Wall }}(\mathrm{z})= \begin{cases}4 \varepsilon\left[\mathrm{U}^{\mathrm{LJ}}(\mathrm{z})-\mathrm{U}^{\mathrm{LJ}}\left(2^{1 / 6} \sigma\right)\right] & \mathrm{z} \prec 2^{1 / 6} \sigma, \\ 0, & \mathrm{z} \succ 2^{1 / 6} \sigma,\end{cases}
$$

where $\mathrm{z}$ is the distance between particles and the wall to which the polymer under consideration is attached. Cutoff distance $\mathrm{zc}$ is set to $2^{1 / 6} \sigma$ to only keep the repulsive interaction. Therefore the total potential energy of the polyelectrolyte brush system is given by

$$
\mathrm{U}_{\mathrm{Total}}=\mathrm{U}_{\mathrm{LJ}}+\mathrm{U}_{\mathrm{FENE}}+\mathrm{U}_{\mathrm{Wall}}+\mathrm{U}_{\mathrm{Coul}}
$$

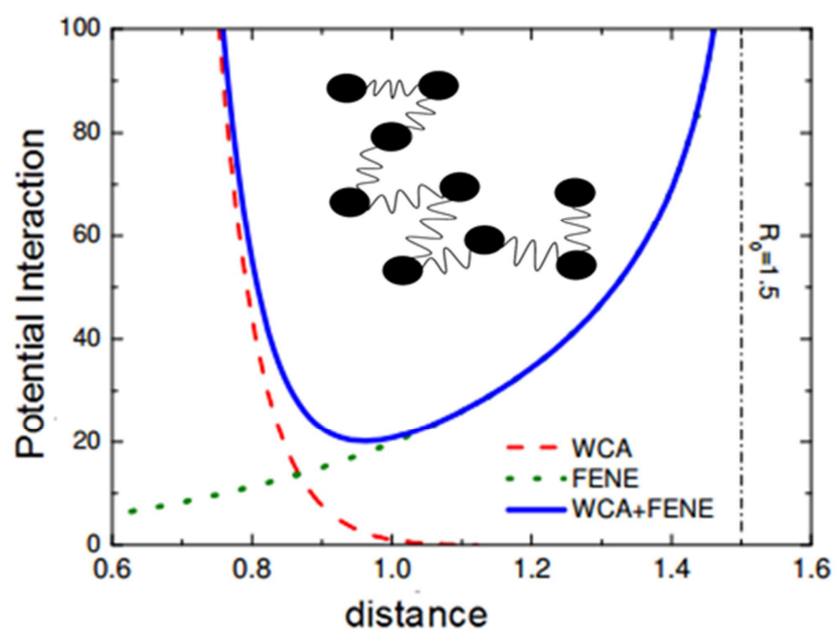

Fig. 7. A comparison of the FENE and WCA potentials, and their sum.

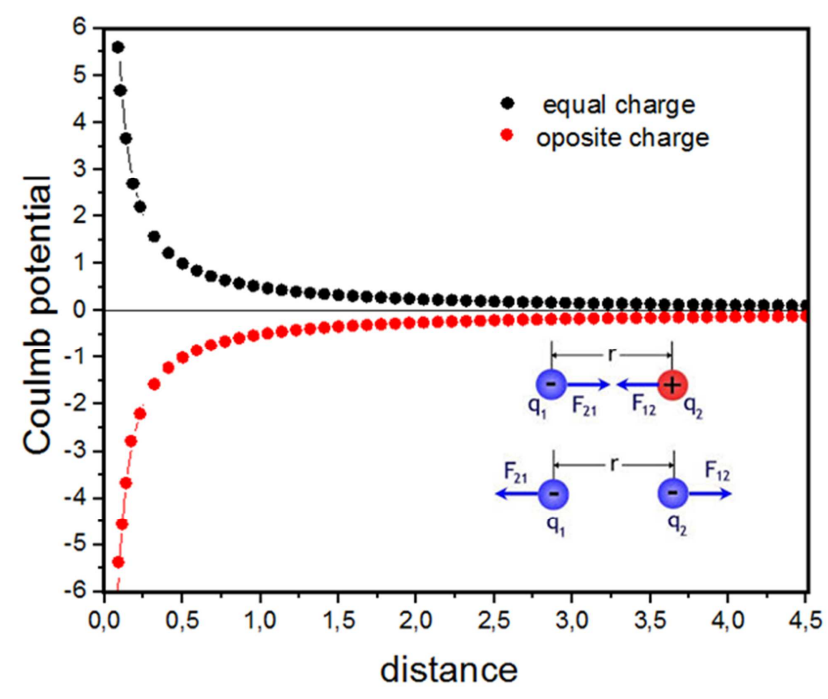

Fig. 8. The Coulomb potential. The bottom curve represents the interaction between two particles of opposite charge, the top curve represents the interaction of particles of equal charge.

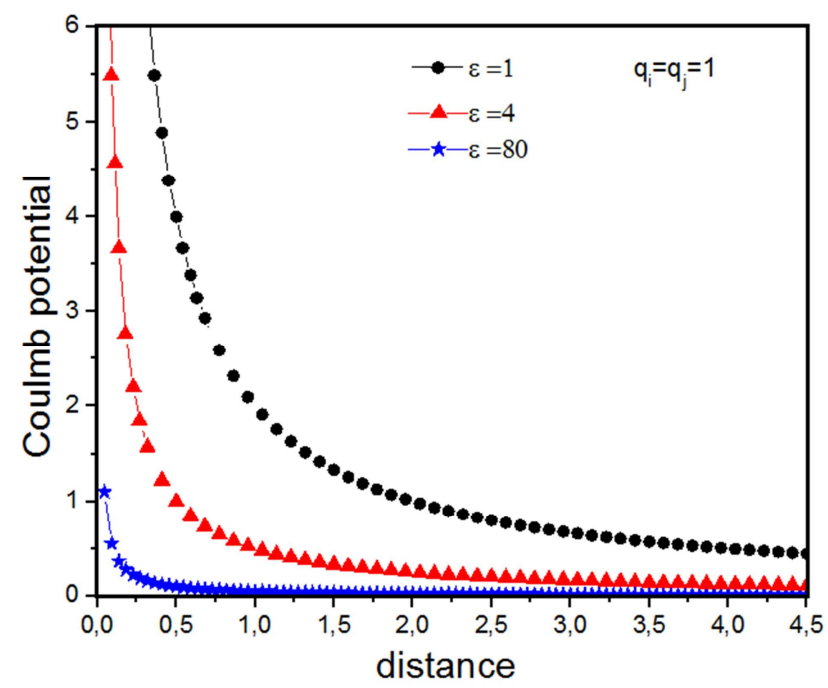

Fig. 9. The Coulomb potential, represents the interaction of the particles of equal load with different values of the effective charge

In classical mechanics, a physical system is described entirely by its potentials and equations of motion (EOM).

The way that MD simulations provide the mechanical properties of the biopolymers is by calculating each monomers' EOM via solving Newton's second law for an interactive many body system numerically. The method used by LAMMPS (Large-scale Atomic/Molecular Massively Parallel Simulator) to evaluate the (EOM) is called the Verlet algorithm There are various, essentially equivalent, versions of the Verlet algorithm, including the original method [25, 26], and a 'leapfrog' form [27]. Here we concentrate on the 'velocity Verlet' algorithm [28], which may be written.

$$
\begin{aligned}
& p_{i}\left(t+\frac{1}{2} \delta t\right)=p_{i}(t)+\frac{1}{2} \delta t f_{i}(t), \\
& r_{i}(t+\delta t)=r_{i}(t)+\delta t p_{i}\left(t+\frac{1}{2} \delta t\right) / m_{i}
\end{aligned}
$$




$$
p_{i}(t+\delta t)=p_{i}\left(t+\frac{1}{2} \delta t\right)+\frac{1}{2} \delta t f_{i}\left(t+\frac{1}{2} \delta t\right)
$$

Notice that the momentum calculated in the first equation goes into the second equation for the evaluation of position. The position enters into the third equation implicitly since the force depends on positions.

In pulling experiments, biopolymers are placed in some solutions with a definite temperature, $\mathrm{T}$. From the derivation of the F-R relation, it is clear that the entropic elasticity can only be manifested when $T \neq 0 \mathrm{~K}$. The motion of particle $\mathrm{i}$ at position $r_{i}(t)$, which is a function of time $t$, is described by Langevin equation, [29]

$$
\mathrm{m} \frac{\mathrm{d}^{2} \mathrm{r}_{\mathrm{i}}}{\mathrm{dt}^{2}}=-\Delta_{\mathrm{i}} \mathrm{U}_{\text {total }}-\mathrm{m} \Gamma \frac{\mathrm{dr}_{\mathrm{i}}}{\mathrm{dt}}+\mathrm{W}_{\mathrm{i}}(\mathrm{t}) .
$$

In this equation, the left side of this equation is the product of mass and the acceleration of particle $i$. The right side is summations of three forces. The first term at the right is the systematic interaction force due to the intra- and intermolecular interaction, which is defined as total potential applied on each particle. The second term is a viscous force (frictional drag) which is proportional to the particle's velocity, where $\Gamma$ is a friction constant. The third term $w_{i}(t)$ is a Gaussian random force, representing the effect of a continuous series of collisions onto the particles from underlying fluid molecules, with

$$
\begin{gathered}
\left\langle\mathrm{W}_{\mathrm{i}}(\mathrm{t}) \mathrm{W}_{\mathrm{i}}\left(\mathrm{t}^{\prime}\right)\right\rangle=6 \mathrm{mk}_{\mathrm{B}} \mathrm{T} \Gamma \delta_{\mathrm{ij}} \delta\left(\mathrm{t}-\mathrm{t}^{\prime}\right), \\
\left\langle\mathrm{W}_{\mathrm{i}}(\mathrm{t})\right\rangle=0,
\end{gathered}
$$

Where \langle\rangle denotes the ensemble's average and $\delta_{i j}$ is the Kronecker delta, and $\Gamma$ is a consequence of the fluctuationdissipation relation. In our simulations.

Table 1. Derived units.

\begin{tabular}{cccc}
\hline$\Gamma$ & T(temperature) & $\tau$ (time) & $\delta \mathrm{t}$ \\
\hline $0.5 \tau^{-1}$ & $1.2 \varepsilon / \mathrm{k}_{\mathrm{B}}$ & $\sigma \sqrt{\mathrm{m} / \varepsilon}$ & $4.510^{5}$ \\
\hline
\end{tabular}

Table 2. Reduced units

\begin{tabular}{cccc}
\hline Mass & Energy & Length & Charge \\
\hline $\mathrm{m}$ & $\varepsilon$ & $\sigma$ & $\mathrm{Q}$ \\
\hline
\end{tabular}

\section{Results and Discussion}

In this section, we presented results of simulations of the variation of friction coefficient as function of velocity for neutral brushes and charged brushes, after we will be interested by numerical studies of the monomer density. We sheared the system by moving the upper wall at constant velocity $(\mathrm{v}, 0,0)$ while keeping the distance between the two surfaces constant. We calculated the normal force $F_{n}$ and shear force Fs directly by adding together the vertical $\mathrm{z}$ components and the horizontal x components, respectively, of forces acting between the substrates and all particles. The corresponding stresses so obtained are labeled $\sigma_{\mathrm{s}}$ and $\sigma_{\mathrm{n}}$, respectively. The friction coefficient is defined as $\mu=\sigma_{\mathrm{s}} / \sigma_{\mathrm{n}}$.

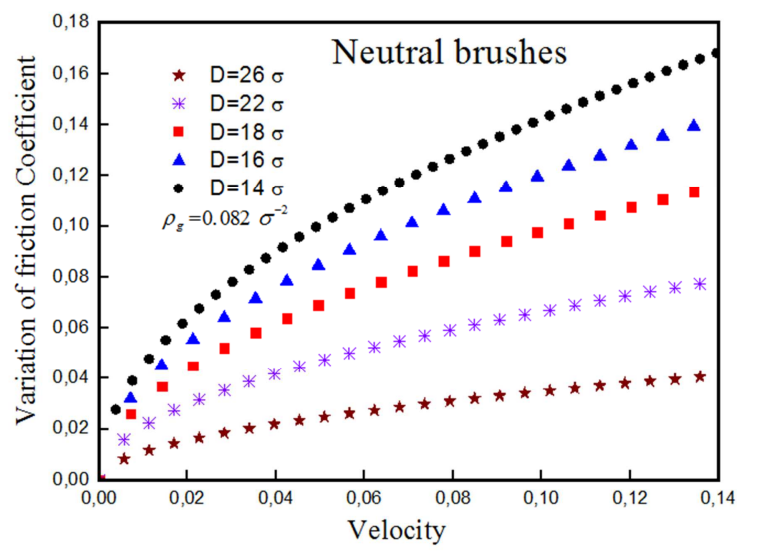

Fig. 10. The variation of friction coefficient as function of velocity for neutral brushes.

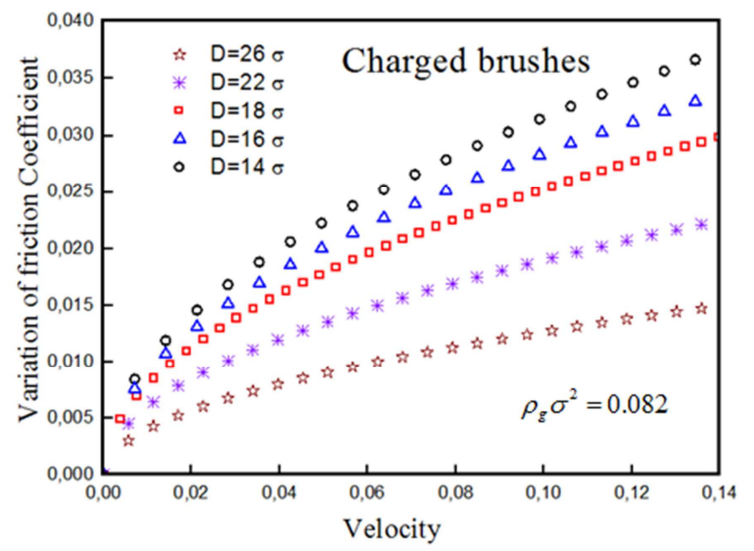

Fig. 11. The variation of friction coefficient as function of velocity for charged brushes.

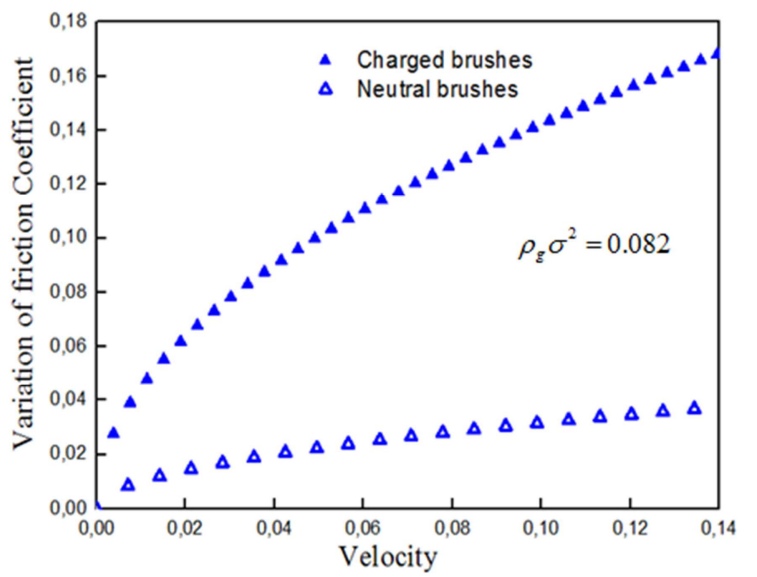

Fig. 12. Comparison between the variation of friction coefficient as function of velocity for neutral and charged brushes

In figure (11), we compare friction coefficients between neutral brushes and polyelectrolyte brushes with parameters specified in the figures captions figure (8), figure (9) and Fig. (10), with $\mathrm{N}=18$ polymer chains of length $\mathrm{N}=36$ 
monomers anchored to the wall surface in a square lattice at the grafting density $\rho_{\mathrm{g}} \sigma^{2}=0.082$.

From our molecular dynamics simulation results, the friction coefficient between neutral polymer brushes is much larger than the value between two charged brushes under same grafting density and surface separation. The normal force was almost constant for both neutral and charged systems as a function of shear velocity. The increase in friction coefficient with velocity is due to the increase in the shear force. When the wall separation distance D was decreased, the normal force and shear force on the wall increased for both neutral and charged brushes. This result comes from stronger interactions between beads in the system because decreasing the surface separation distance D results in an increase in the global monomer density of the polymer brushes, which can be seen in figure (12), and the counterion density. Also, the values of normal forces on the walls in the polyelectrolyte brush systems are about an order of magnitude greater than the values in the neutral brushes, even though both of them have the same grafting density and chain length.

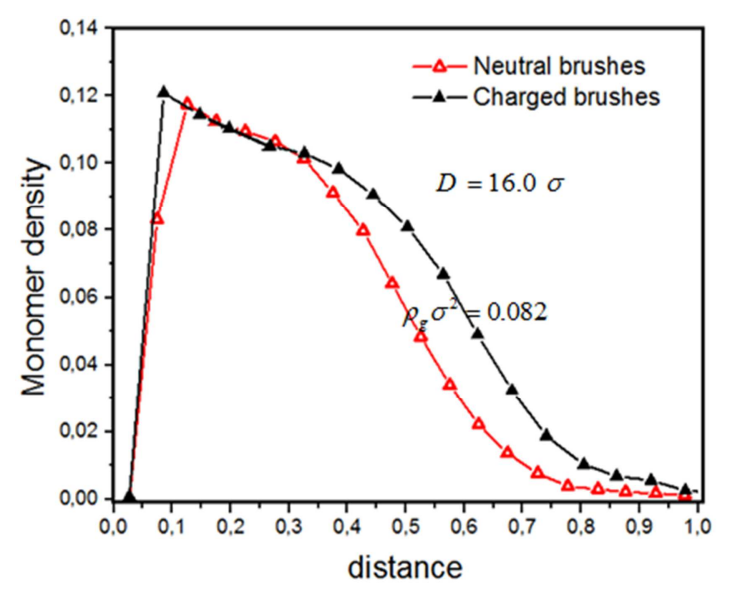

Fig. 13. The monomer density profiles for neutral brushes (Red line with dotted triangles) and charged brushes (Black line with solid triangles) without the relative sliding. With separation distance

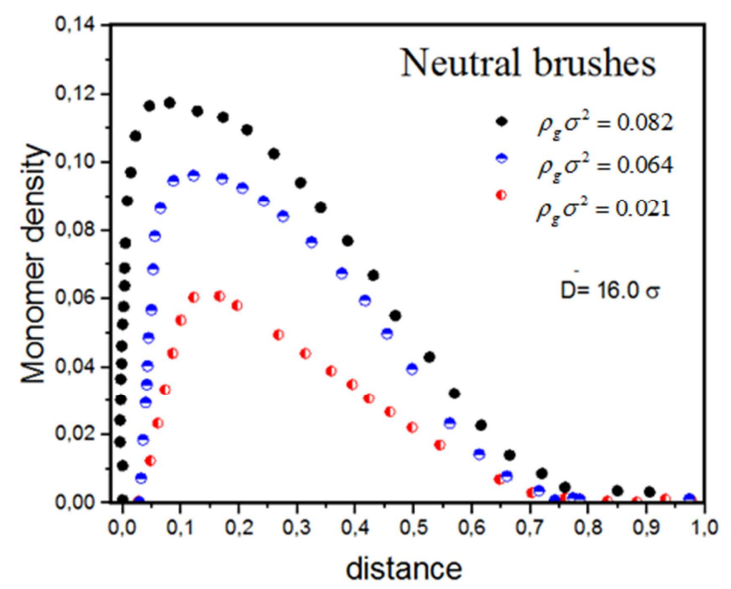

Fig. 14. The monomer density with separation distance and three values of the grafting density of polymer brushes, (Blue dotted circles), (Blue dotted circles).

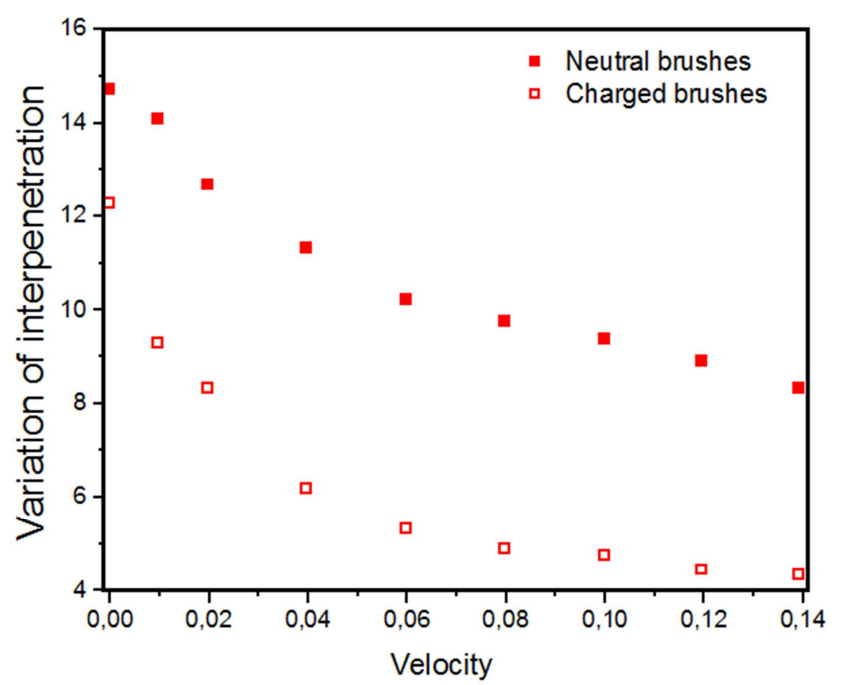

Fig. 15. Comparison between the variation interpenetration as function of velocity for neutral and charged brushes (Red dotted circles).

\section{Conclusion}

Using the MD simulations, we simulate the relative shear motion of both neutral and polyelectrolyte end-grafted polymer brushes on two parallel membranes, which are immersed in a solvent. From our molecular dynamics simulations, for the case of equal grafting density, we demonstrated that polyelectrolyte brushes have a smaller friction coefficient (order of magnitude)and monomer penetration than neutral polymer brushes, although the polyelectrolyte brushes supported a much higher normal load than the neutral brushes for the same degree of compression. Charged and neutral brushes with their grafting densities chosen so that they support the same load exhibited approximately the same degree of interpenetration, but the polyelectrolyte brush exhibited a significantly lower friction coefficient. For these systems, the normal force is much larger in the charged system and the number of contacts is smaller in the charged system. Our results suggest that the extra normal force contribution provided by the counter ion osmotic pressure that exists for polyelectrolyte brushes permits polyelectrolyte brushes to support the same load as an identical neutral polymer brush of higher grafting density. Because of the resulting lower monomer density for the charged brushes, fewer monomer collisions take place per unit time, resulting in a lower friction coefficient. Surprisingly, the interpenetrations between top and bottom brushes of both neutral brushes and charged brushes are almost same when we exert the same normal force pressure on the grafting membrane surfaces of these two kinds of brushes.

\section{Author Contributions}

Both authors contributed equally to the development of this paper. 


\section{Acknowledgments}

The authors are thankful to Prof. M. Ouarch for his valuable remarks and discussion, which made it possible to reevaluate the work from another view- point.

\section{References}

[1] B. Alberts, D. Bray, J. Lewis, M. Raff, K. Roberts, and J. D. Watson, "Molecular Biology of the Cell," Garland, New York, (1944).

[2] E. Sackmann, "Membrane bending energy concept of vesicleand cell-shapes and shape-transitions," FEBS Letters, 346 (1994) 3 .

[3] R. Lipowsky and S. Leibler, "Unbinding transitions of interacting membranes," Physical Review Letters, 56 (1986) 2541 .

[4] U. Seifert and R. Lipowsky, "Adhesion of vesicles," Physical Review A, 42 (1990) 4768.

[5] G. I. Bell, "Models for the specific adhesion of cells to cells," Science, 200 (1978) 618.

[6] G. I. Bell, M. Dembo, and P. Bongrand, "Cell adhesion. Competition between nonspecific repulsion and specific bonding, Biophysical Journal, 45 (1984) 1051.

[7] E. A. Evans, "Detailed mechanics of membrane-membrane adhesion and separation. I. Continuum of molecular crossbridges," Biophysical Journal, 48 (1985) 175.

[8] Matthias Ballauff and Oleg Borisov. Polyelectrolyte brushes.

[9] Current Opinion in Colloid Interface Science, 11 (2006) 316.

[10] JurgenRuhe et al. Polyelectrolyte brushes. In Polyelectrolytes with Defined Molecular Architecture I, Advances in Polymer Science, 165 (2004) 189.

[11] Gary S. Grest. Interfacial sliding of polymer brushes: A molecular dynamics simulation. Phys. Rev. Lett., 76 (1996) 4979.

[12] Michael Murat and Gary S. Grest. Interaction between grafted polymeric brushes: A molecular-dynamics study. Phys. Rev. Lett., 63 (1989) 1074.

[13] T. Kreer, M. H. Muser, K. Binder, and J. Klein. Frictional drag mechanisms between polymer-bearing surfaces. Langmuir, 17 (2001) 7804.

[14] Daniel J Sandberg, Jan-Michael Y Carrillo, and Andrey V Dobrynin. Molecular dynamics simulations of polyelectrolyte brushes: from single chains to bundles of chains. Langmuir: the ACS journal of surfaces and colloids, 23 (2007) 12716.

[15] Owen J Hehmeyer and Mark J Stevens. Molecular dynamics simulations of grafted polyelectrolytes on twoapposing walls. The Journal of chemical physics, 122 (2005) 134909.
[16] Qianqian Cao, Chuncheng Zuo, Lujuan Li, and Hongwei He. Shearing and compression behavior of end-grafted polyelectrolyte brushes with mono- and trivalent counterions: a molecular dynamics simulation. Modelling and Simulation in Materials Science and Engineering, 18 (2010) 075001.

[17] J B Sokoloff. Theory of the observed ultralow friction between Sliding polyelectrolyte brushes. The Journal of chemical physics, 129 (2008) 014901.

[18] Jacob Klein, Kumacheva Eugenia, Perahia Dvora, and Mahalu Diana. Shear, friction, and lubrication forces between polymer bearing surfaces. Annual Reviews of Material Science, 26 (1996) 581

[19] Allen, M. P., Introduction to Molecular Dynamics Simulation, published in NIC Series (2004) Vol. 23, 1-8.

[20] Weeks, J. D.; Chandler, D.; Andersen, H. C. Role of repulsive forces in determining the equilibrium structure of simple liquids. J. Chem. Phys. 54(1971) 5237.

[21] Grest, G. S.; Kremer, K. Molecular dynamics simulation for polymers in the presence of a heat bath. Phys. Rev. A 33, (1986), 3628.

[22] Kremer, K.; Grest, G. S. Dynamics of entangled linear polymer melts: A molecular-dynamics simulation. J. Chem. Phys. 92, (1990) 5057.

[23] Kurt Kremer and G. S. Grest. Molecular-dynamics of entangled linear polymer melts: An simulation. The Journal of Chemical Physics, 92(1990)5057.

[24] Peter A. Thompson, Gary S. Grest, and Mark O. Robbins. Phase transitions and universal dynamics in confined films. Phys. Rev. Lett, 68(1992) 3448.

[25] Lyubimov, I.; Guenza, M. G. First-principle approach to rescale the dynamics of simulated coarse-grained macromolecular liquids. Phys. Rev. E (2011), 84, 031801:1031801:19.

[26] L. Verlet. Computer experiments on classical_uids. i. thermodynamical properties of Lennard-Jones molecules. Phys. Rev, 159(1967) 98-103.

[27] L. Verlet. Computer experiments on classical _uids. ii. equilibrium correlation functions. Phys. Rev., 165(1968)201214 .

[28] R. W. Hockney and J. W. Eastwood. Computer simulations using particles. Adam Hilger, Bristol, (1988).

[29] W. C. Swope, H. C. Andersen, P. H. Berens, and K. R. Wilson. A computer simulation method for the calculation of equilibrium constants for the formation of physical clusters of molecules: application to small water clusters. J. Chem. Phys., 76(1982)637-649.

[30] F. Reif, Fundamentals of Statistical and Thermal physics (McGraw-Hill, New York, (1965)). 